\title{
Hormone treatment of gender identity disorder in a cohort of children and adolescents
}

\section{Jacqueline K Hewitt MB BS, FRACP, Fellow and PhD Scholar Department of Endocrinolog and Diabetes ${ }^{1}$}

Campbell Pau MB BS, FRANZCP Psychiatrist $^{1}$

Porpavai Kasiannan DPM, MD, FRANZCP Psychiatrist

Sonia R Grove MB BS, MD, FRANZCOG Gynaecologist

Louise K Newman MB BS, PhD, FRANZCP, Psychiatrist $^{2}$

Garry L Warne MB BS, FRACP, Honorary Physician in Endocrinology

I Royal Children's Hospital, Melbourne, VIC

2 Centre for Developmental Psychiatry and Psychology,

Monash Medical Centre Melbourne, VIC.

jacky.hewitt@rch.org.au

MJA 2012; 196: 578-58 doi: 10.5694/mjal2.10222 ndividuals with gender identity disorder (GID) have a persistent and profound discomfort with their biological sex and a strong identification with the gender of the opposite sex. The diagnosis can be made when this is manifested by unrelenting crossgender thought and behaviour. ${ }^{1,2}$

Children as young as 3 years of age may experience gender dysphoria and cross-gender behaviour; however, only $16 \%$ of these children will continue to have persistent GID in adolescence and adulthood. ${ }^{3}$ In the group with profound and persisting cross-gender identification it is characteristic for severe distress to be exacerbated at the onset of puberty, due to revulsion toward unwanted physical changes. Of the larger group of children who do not continue to have persistent GID in adolescence and adulthood, it is reported that $42 \%$ will eventually identify as homosexual or bisexual. ${ }^{4}$

In addition to ongoing psychological support, peripubertal adolescents with persistent GID may be given hormonal treatment using gonadotrophin-releasing hormone $(\mathrm{GnRH})$ analogue to suppress puberty once it has commenced, followed later by cross-sex hormone therapy to promote physical development in the affirmed gender. ${ }^{5}$ Current evidence suggests that when a strict assessment and clinical protocol is followed, hormone treatment to suspend the development of puberty is associated with a good outcome and a low rate of regret. ${ }^{3,6-8}$

The rationale for hormone treatment to suppress puberty is to reduce the psychological distress associated with unwanted pubertal development in the biological sex (which can result in hormonal self-medication, selfharm or suicide). ${ }^{9}$ This allows additional time for continued psychological

\section{Abstrac \\ Objective: To describe the experience of hormone treatment of gender identity} disorder (GID) in children and adolescents within a specialist clinic.

Design, patients and setting: Cohort study by medical record review of children aged 0-17 years referred during 2003-2011 for management at the GID clinic in a tertiary paediatric referral centre - the Royal Children's Hospital, Melbourne, Victoria.

Main outcome measures: Clinical characteristics of the patient population, hormone treatment provided, frequency of referrals with time.

Results: Thirty-nine children and adolescents were referred for gender dysphoria. Seventeen individuals were pubertal with persistent GID, and were considered eligible for hormone treatment. Seven patients, comprising three biological males and four biological females, had legally endorsed hormone treatment. In this group, gender dysphoria was first noted at 3-6 years of age. Hormone treatment with $\mathrm{GnRH}$ analogue to suppress pubertal progression (phase 1) was given at 10-16 years of age. Treatment with cross-sex hormones (phase 2) was given at 15.6-16 years. One patient purchased cross-sex hormone treatment overseas. One patient received oestrogen and progesterone for menstrual suppression before phase 1. The annual frequency of new referrals increased continuously over the study period.

Conclusions: Hormone treatment for pubertal suppression and subsequent gender transition needs to be individualised within stringent protocols in multidisciplinary specialist units.

support during the process of either resolving gender dysphoria or coping with its persistence, and to prevent irreversible secondary sex changes, which may be difficult to address later on and may significantly compromise adjustment in adulthood.

Specialist clinics for child and adolescent GID have been established in Canada, the United States, the United Kingdom and several European countries. International consensus guidelines for hormonal treatment of adolescents with GID have been published $^{10}$ and subsequently endorsed by the Australasian Paediatric Endocrine Group; ${ }^{11}$ and standards of care for children and adolescents with GID have also been published. ${ }^{12}$ At present, authorisation from the Family Court of Australia is required before commencement of hormone treatment leading toward gender reassignment in patients aged under 18 years. Past legal judgments have defined this treatment a "special medical procedure", whereby parents are not able to consent to treatment on behalf of a child and the court acts to ensure valid informed consent. Australia is the only country with this requirement, and the legal costs incurred in seeking medical treatment of GID in an adolescent are borne by the family.

Over the past 10 years, the number of children with gender dysphoria referred to the Royal Children's Hospital Melbourne (RCHM) has steadily increased. To provide a more comprehensive treatment program in Victoria, an interdisciplinary clinical group was formed in collaboration with Monash Medical Centre. The process for psychological and medical treatment of children and adolescents with GID established in Melbourne accords with international guidelines (Box 1). ${ }^{10-13}$

We reviewed the experience of hormone treatment of GID in children 
1 Current procedure for treating children and adolescents with GID who are referred to the RCHM Gender Identity Service

- Parents who suspect their child is experiencing gender dysphoria can seek help from a range of health or educational professionals, who may then refer to mental health clinicians within the RCHM Gender Identity Service.

- A full psychiatric assessment follows, with the aim of engaging the child or adolescent in ongoing psychotherapy. For young adolescents entering puberty with profound and persisting GID, there may be discussions about the possibility of delaying progression of puberty. The current procedure, consistent with published protocol, ${ }^{13}$ involves two independent child and adolescent psychiatrists undertaking a standardised assessment of psychological development, and a formal assessment of the child's gender identification and capacity to understand any proposed treatment.

- A formal diagnosis of persistent GID may be made if the young person meets the diagnostic criteria of the Diagnostic and statistical manual of mental disorders, fourth edition.

- A paediatric endocrinologist establishes pubertal staging, excludes disorders of sex development, and counsels regarding the reversible and irreversible effects and risks of hormonal treatment in eligible patients. Patient handouts and consent forms have been developed to assist with this (Box 2).

- Fertility counselling is provided by a gynaecologist or andrologist before application for clinical ethics review and Family Court authorisation of treatment. Phase 1 hormone treatment to suppress puberty can occur from Tanner pubertal stage $2-3$. Phase 2 cross-gender hormone treatment can be given from age 16 years.

- Surgery is not considered in patients aged less than 18 years and is only offered after transition to adult care providers.

$\mathrm{GID}=$ gender identity disorder. RCHM = Royal Children's Hospital Melbourne.

and adolescents at RCHM over the 8year period since it commenced in 2003. In particular, we aimed to outline the clinical characteristics of the patient population, the treatment provided and frequency of referrals over time.

\section{Methods}

Children and adolescents referred to RCHM for gender dysphoria during 2003-2011 were included in the study. Patient medical records were reviewed. Data were collected for gender transition, age at commencement of gender dysphoria, age at referral, date of referral, family and medical history, hormone treatment eligibility, treatment application and outcome, treatment prescribed, age at treatment commencement and duration of follow-up.

All patients were assessed by a child and adolescent psychiatrist using standard procedure and regular psychiatric review.

Records of the Family Court of Australia were searched via the Australasian Legal Information Institute database for judgments related to special medical procedures, and recorded cases were verified against the study cohort.

The study was approved by the $\mathrm{RCH}$ Human Research Ethics Committee.

\section{Results}

During 2003 to 2011, 39 children and adolescents were referred to the service and assessed. Of these, 18 patients were pre-adolescent and continued to have ongoing developmental psychological counselling. Twenty-one were either approaching puberty or pubertal, and were referred to a second psychiatrist for formal confirmation of a diagnosis of GID. Adolescent patients with persistent GID were then assessed by a paediatric endocrinologist. The adolescent group comprised 13 biological males and eight biological females.

In the group of 21 adolescents reviewed for consideration of hormone treatment, the mean age at which GID was first perceived to be a problem was $3.26 \pm 1.11$ years. The mean age at presentation for GID assessment to any member of the service was $10.0 \pm 4.13$ years. Two of the adolescents were from dizygotic same-sex twin sibships, discordant for GID.

During independent psychiatric evaluation, all 21 adolescents reported symptoms of anxiety or depression. Suicidal ideation was also reported. Many children expressed an intense sense of revulsion towards parts of their bodies and wished to have their genitals cut or modified. Other comorbid psychiatric disorders were present in five children. These included obsessional disorders in two, and Asperger syndrome in three, including patients of both biological sexes. There was a reported family history of homosexuality or gender dysphoria in first- or second-degree relatives in four of 12 children for whom detailed family history data were recorded.

Of the 21 adolescents reviewed for consideration for hormone treatment, four experienced resolution of gender dysphoria or acceptance of gender variance with ongoing psychological counselling. Seventeen adolescents experienced persistence of profound GID with increased distress following commencement of puberty and were therefore considered eligible for hormone treatment. In this subset of eligible patients, 11 had made or planned to make an application to the Family Court for hormone treatment. As the families are required to themselves apply for court approval, in all cases the child was supported in their application by at least one parent. Reasons for not seeking court-approved hormone treatment included: age close to 18 years (when court approval is not required) in two cases, court financial cost in two cases, family not supporting hormone treatment in one case and one affirmed female patient who purchased oestrogen independently overseas without court approval.

Seven patients (three biological males and four biological females) commenced court-approved hormone treatment. In this group, gender dysphoria was first noted at 3-6 years of age. Treatment with $\mathrm{GnRH}$ analogue to suppress pubertal progression (phase 1) was started at 10-16 years of age. Cross-sex hormones (phase 2) were introduced at $15.6-16$ years.

Four patients received both $\mathrm{GnRH}$ analogue and cross-sex hormones, two had GnRH analogue alone and two had only cross-sex hormones. One patient also received continuous oral oestrogen and progesterone followed by depot medroxyprogesterone acetate for menstrual suppression before phase 1 treatment with $\mathrm{GnRH}$ analogue. Five patients commenced medical intervention before completion of puberty in their biological sex. All patients who had phase 1 treatment went on to have phase 2 therapy when eligible. 
2 Effects of hormone treatment of GID in adolescence*

Continuous oestrogen/ progesterone (females with gender dysphoria)

- Suppression of menses

GnRH analogue (MTF and FTM)

- Slower bone density accrual with catch-up at pubertal induction

- Slower growth with catch-up at pubertal induction

Oestrogen (MTF)

- Breast development and feminine body habitus

- Testicular softening and shrinkage

- Growth plate maturation with possible final height reduction

- Effects on mood, migraine headache, liver function, prolactin level and thromboembolism risk

Testosterone (FTM)

- Hair growth

- Acne

- Muscle bulk increase

- Voice deepening (irreversible)

- Clitoral growth and increased erections

- Reduction or suppression of menses

- Growth plate maturation with possible final height reduction

- Effects on mood, erythrocytosis, lipid profile, liver function, polycystic ovaries, possible endometrial or ovarian cancer

* This information is provided to patients, families, and the Family Court of Australia before any hormone treatment is commenced. $\mathrm{GnRH}=$ gonadotrophin-releasing hormone. $\mathrm{MTF}=$ male to female. $\mathrm{FTM}=$ female to male.

A search of the records of the Family Court of Australia found no additional cases of application for hormone treatment for GID.

Our follow-up experience for adolescents undergoing hormone treatment for GID is 20.7 person-years (range $0-8.2$ years). No patient has ceased hormone treatment.

The annual frequency of referrals increased eightfold from one patient in 2003 to eight patients in 2011.

\section{Discussion}

Our review showed that while crossgender identification was noted in all patients from about 3 years of age, children did not present to our specialist service until a mean age of 10 years. Many families reported difficulty in finding specialists in this field; some medical and paramedical clinicians had previously rejected these patients from care. Our experience was that patients and their families were relieved to find specialist health care providers for paediatric GID. Of the patients considered eligible for hormone treatment, many wished to commence treatment urgently, with the court approving phase 2 treatment in one child at 15.6 years (younger than the current guideline of 16 years).

The number of referrals increased over the study period and, based on international experience, ${ }^{14}$ is expected to rise further. We believe this increase is due to improved awareness of a medical service for children with GID, rather than any increase in the incidence of the disorder. In addition to the increasing numbers of new referrals, more than half of our patients are yet to reach puberty. Development of expertise in this area, including good transitional services to adult care, is necessary.

There have been a number of challenges involved in establishing a treatment service for children with GID, from administrative concerns such as the gender and name assigned on the medical record, to comorbid diagnosis concerns, restrictions on Pharmaceutical Benefits Scheme funding for hormone therapy, and appropriate choice of treatment dependent on physical and mental developmental stage. For example, GnRH analogue treatment, when used to suppress pubertal progression in a distressed peripubertal young person, provides more useful time for psychological counselling, but in a postpubertal biological female, simple suppression of menses using continuous treatment with the oral contraceptive pill may be more appropriate. While a clear management protocol has been developed, much consideration is required for each individual case.

Due to the specialised nature of assessment and treatment, most cases nationally have been referred to $\mathrm{RCHM}$ when hormone treatment is considered. To the best of our knowledge, adolescents who received hormone treatment at RCHM during the period studied represent the entire population of children receiving puberty suppression and cross-gender hormone treatment for GID in Australia.

Psychological causations have been proposed, however the precise aetiol- ogy of profound GID is not known. Biological factors may also be significant in causation of childhood GID, and a heritable component has been suggested by twin association studies. ${ }^{15,16}$ We noted comorbid behavioural disorders in one-quarter of children, with Asperger syndrome in one in seven. An association between paediatric GID and autism spectrum disorders has previously been reported, however the mechanism is also unknown. ${ }^{17,18}$

Concerns exist regarding the longterm outcome following hormone treatment of children and adolescents with GID. ${ }^{19}$ Evidence from larger international cohorts suggests behavioural problems and depression improve in the period following pubertal suppression, but anxiety, anger, and gender dysphoria may remain unchanged. ${ }^{8}$ The long-term psychological and health outcomes of cross-sex hormone treatment are unknown, as is the rate of "regret" with reversal of gender identity.

These concerns, along with issues relating to the ability of a child or his or her parents to consent to such significant medical treatment, contribute to legal involvement in medical decision making in this area. The unfortunate corollary to this is that, as the financial burden of legal involvement is borne by the family and the process itself can be extremely stressful, some families have elected not to pursue hormone treatment, simply to avoid the complicated and costly legal process. These do not seem equitable or just reasons for restricting a young person's access to medical care. Furthermore, a young person observing the distress that court application causes for the parents may feel uncomfortable expressing any doubts they have regarding hormone treatment.

There is a growing body of literature on the management of GID in children, including internationally established consensus treatment guidelines, and keeping abreast of the scientific literature is a priority in clinical management. Because data on long-term outcomes are limited, assessment and treatment must be rigorous. We suggest that treatment should occur within specialised clinics where there are established clinical protocols in place, in a shared care 
arrangement with clinicians at remote sites if required. In practice, very close collaboration is required between psychiatrists, endocrinologists and gynaecologists, with regular case conferencing and clinical group meetings to discuss management, establish and audit process, and review new literature. Consultation with legal counsel and clinical ethicists is also required.

Providing treatment to suspend the development of normal puberty may present an ethical dilemma for some physicians. Due to the complexity of treatment, we have established a stringent process of assessment and management within a clinical service for children with GID, and details can be found at http://www.rch.org.au/ outpatient/directory/index.cfm?fuseaction=home.full\&id=127.

The response of clinicians to children with gender dysphoria has been varied, with some specialist practitioners who are willing to provide medical care encountering unclear referral and management pathways. We support the establishment of specialised, formal paediatric GID services within tertiary paediatric centres elsewhere in Australia, according to similar protocols and guidelines.

The experience of growing up in a body which feels alien is difficult and confusing. Profound GID represents a mental health crisis in childhood, with implications for problematic psychosocial and relationship develop- ment throughout life. Our experience is consistent with reports from international centres - that children and adolescents with GID suffer with a great burden of morbidity, and are deserving of optimal medical care. However, in view of the significance of hormonal treatment and the still emerging evidence base, rigorous assessment and follow-up is essential, along with the stringent auditing and publication of outcomes.

Competing interests: No relevant disclosures.

Received 3 Feb 2012, accepted 12 Apr 2012.

1 American Psychiatric Association. Diagnostic and statistical manual of mental disorders. 4th ed. Washington: American Psychiatric Publishing, 2000.

2 World Health Organization. The ICD-10 classification of mental and behavioural disorders. Geneva: World Health Organization, 1992.

3 Steensma TD, Biemond R, Boer FD, CohenKettenis PT. Desisting and persisting gender dysphoria after childhood: A qualitative followup study. Clin Child Psychol Psychiatry 2011; 16: 499-516.

4 Wallien MSC, Cohen-Kettenis PT. Psychosexual outcome of gender-dysphoric children. J Am Acad Child Adolesc Psychiatry 2008; 47: 1413-1423.

5 Gooren LJ. Clinical practice. Care of transsexual persons. N Engl J Med 2011; 364: 1251-1257.

6 Delemarre-van de Waal HA, Cohen-Kettenis PT. Clinical management of gender identity disorder in adolescents: a protocol on psychological and paediatric endocrinology aspects. Eur J Endocrinol 2006; 155: SI31-SI37.

7 Kreukels BPC, Cohen-Kettenis PT. Puberty suppression in gender identity disorder: the Amsterdam experience. Nat Rev Endocrinol 2011; 7: 466-472.

8 Cohen-Kettenis PT, Schagen SEE, Steensma TD, et al. Puberty suppression in a gender-dysphoric adolescent: a 22-year follow-up. Arch Sex Behav 2011; 40: 843-847.
9 de Vries ALC, Steensma TD, Doreleijers TAH, Cohen-Kettenis PT. Puberty suppression in adolescents with gender identity disorder: a prospective follow-up study. J Sex Med 2011; 8: 2276-2283.

10 Cohen-Kettenis PT, Delemarre-van de Waal HA, Gooren LJG. The treatment of adolescent transsexuals: changing insights. J Sex Med 2008 5: 1892-1897.

11 Hembree WC, Cohen-Kettenis P, Delemarre-van de Waal HA, et al; Endocrine Society. Endocrine treatment of transsexual persons: an Endocrine Society clinical practice guideline. J Clin Endocrinol Metab 2009; 94: 3132-3154.

12 Australasian Paediatric Endocrine Group Disorders of Sex Development Working Group. Position statement and management guidelines. Gender Identity Disorder Guidelines. 2010. http://www.apeg.org.au/ PositionStatementManagementGuidelines/ tabid/87/Default.aspx (accessed Apr 2012).

13 World Professional Association for Transgender Health. Standards of care for the health of transsexual, transgender, and gender nonconforming people. 7th version. 2011. http://www.wpath.org/publications standards.cfm (accessed Apr 2012).

14 Coolidge FL, Thede LL, Young SE. The heritability of gender identity disorder in a child and adolescent twin sample. Behav Genet 2002; 32: 251-257.

15 Heylens G, De Cuypere G, Zucker, KJ, et al. Gender identity disorder in twins: a review of the case report literature. J Sex Med 2012; 9: 751-757.

16 de Vries A, Noens I, Cohen-Kettenis P. Autism spectrum disorders in gender dysphoric children and adolescents. J Autism Dev Disord 2010; 40: 930-936.

17 Kraemer B, Delsignore A, Gundelfinger R, et al. Comorbidity of Asperger syndrome and gender identity disorder. Eur Child Adolesc Psychiatry 2005; 14: 292-296.

18 Spack NP, Edwards-Leeper L, Feldman HA, et al. Children and adolescents with gender identity disorder referred to a pediatric medical center. Pediatrics 2012; 129: 418-425.

19 Korte A, Lehmkuhl U, Goecker D, et al. Gender identity disorders in childhood and adolescence: currently debated concepts and treatment strategies. Dtsch Arztebl Int 2008; 105: 834-841. 